\section{P-49 ALL THINGS BEING EQUAL - IMPROVING OUR APPROACH TOWARDS EQUALITY, DIVERSITY AND INCLUSION (EDI)}

Alex Taylor. St Wilfrid's Hospice, Eastbourne, UK

10.1136/bmjspcare-2018-hospiceabs.74

Background Equality and diversity should be integrated into palliative care (Care Quality Commission, 2016). Awareness of our patients' unique demographics helps individualise their care. Discrimination and assumptions should be avoided (Marie Curie, 2016; Baillie \& Matiti, 2012). Fear of this may inhibit certain groups from accessing care. Analysis of previous patient data identified certain demographics, particularly religious beliefs and sexual orientation, were consistently poorly recorded.

Aims Evaluate staff beliefs, knowledge and skills surrounding equality, diversity and inclusion (EDI) in the organisation. Use results to shape staff training.

Method Online anonymised survey sent to all staff via email. Areas covered include staff role, importance of EDI to practice, how often they ask patients about disability, religious beliefs, ethnicity, gender identity, sexual orientation, comfort in doing so and whether the hospice encourages a culture of EDI.

Results 62 responses:

- 33 nurses, seven doctors, five therapists, 17 non-clinical

- $100 \%$ rated awareness of disability and beliefs as important to their role, $93 \%$ ethnicity and gender identity, $83 \%$ sexual orientation.

- $69 \%$ of staff do not ask about sexual orientation, 64\% do not ask about gender identity.

- Most common reason for not asking: 'fear of making patient uncomfortable' $-23 \%$ of staff chose this for sexual orientation and gender identity.

- Majority preferred to seek information by asking patients directly but high number (33\% for ethnicity and sexual orientation) stated they made assumptions based on patient interaction.

- $80 \%$ felt face to face best to collect this information, $58 \%$ thought form would be suitable.

- Asked whether organisation encourages culture of EDI for patients $-8.7 \%$ of staff responded 'no', 32.6\% 'unsure'. 22\% 'unsure' whether EDI encouraged amongst staff.

Conclusions Findings clearly demonstrate work needs to be done to promote EDI in the hospice. EDI has now been incorporated into all staff's mandatory training. New working group set up, seeking to promote EDI and increase accessibility to less represented groups.

\section{P-50 RUNNING A DEATH CAFÉ - PROMOTION, PLACE, PARTICIPANTS AND PRODUCT}

Sarah Bell. Garden House Hospice Care, Letchworth Garden City, UK

\subsection{6/bmjspcare-2018-hospiceabs.75}

Background The principles of running a death café are widely available (Morgan, 2017; Baldwin, 2017) but information comparing different styles, venues or promotion of cafés is unobtainable. Published Death Café model guidance advises against professionally experienced end of life care facilitators (http://deathcafe.com/how/).

Aims To share learning from three different death cafés regarding promotion, title, venues, facilitators and participants. Method Three death cafés were run: two public and one for staff. All had clinical hospice staff as facilitators.

The 2015 public event was entitled 'Death Café'. The venue was a suburban church hall. There were simple refreshments. The host church helped with local promotion and event organisation.

The 2018 public event was promoted by the hospice locally and via media. It was entitled 'Everything You Wanted to Know About Dying but Were Afraid to Ask'. Drinks and cake were provided. The event was at a town edge church venue, challenging to locate. No host church involvement.

The 2018 internal event, aimed at hospice volunteers and non-clinical staff, was entitled 'Death Café'. The venue was the main hospice building. Promotion was internal.

Results Attendance at the first and second external events was 40 and 12 respectively. Many first event participants were from the host church. Both events had participants from faith groups but no 'passing trade' attendees. Both venues were free. Participants easily located the suburban but not the town edge venue. The internal event was attended by 16 staff and volunteers. No staff working outside the main hospice site attended. For all events, clinical hospice facilitators were clearly beneficial in addressing participants' fears and misinformation.

Conclusion The title Death Café does not deter attendees. Easily located venues are essential. Advertising to local faith groups is worthwhile. Fancy refreshments are unnecessary. Hosting by a faith group can reduce workload, increases attendees and reduce cost. Hospice staff are less likely to attend events outside their usual workplace. Clinically experienced facilitators are beneficial.

\section{P-51 DEATH CAFÉS: OPENING UP ABOUT DEATH, DYING AND BEREAVEMENT}

Mhairi Herring, Hayley Purser. Ashgate Hospicecare, Chesterfield, UK

\subsection{6/bmjspcare-2018-hospiceabs.76}

Background Talking about death and dying is a tough subject, however, it is something that is going to happen to all of us. The concept of death cafés was developed by Jon Underwood and Sue Barsky Reid in 2011 to provide a secure place to talk openly about death, dying and bereavement and to help people make the most of their finite lives (http://deathcafe. com; Miles \& Corr, 2017).

Aims Ashgate Hospicecare wants to build on the death café movement and take away the taboo of a subject that we all face. We also aim to use these events to tackle the misconceptions surrounding hospice culture.

Method In 2016, Ashgate Hospicecare's first death café was held in a local library with support from solicitors and funeral directors. By 2018, the event was held at a unit in the town centre over two days and within the hospice for one day. Each year, more interactive opportunities were available for the general public and hospice staff than the previous year, such as creating memory boxes, finger print keyrings, thinking about your funeral song and creating a bucket list. We have 
measured the success of the cafés by recording attendance rates and through conducting qualitative surveys.

Results Attendance rates: 2016: 19, 2017: 43, 2018: 82.

Conclusion During the three years of death cafés at Ashgate Hospicecare, there has been an evident growth in attendance rates. To complement this, the surveys have shown more positive feedback from death café attendees and growing interest from outside organisations who want to be involved in future events. Moving forward, Ashgate Hospicecare aims to run death cafés throughout the year, working in partnership with other community groups, such as schools and clinical organisations. We strongly believe in the importance of raising awareness around death and dying and helping people plan for their future.

\section{P-52 LEARNING FROM BEFRIENDERS IN COMPASSIONATE COMMUNITIES - SERVICE CO-PRODUCTION WITH VOLUNTEERS}

Tracy Livingstone. Nightingale House Hospice, Wrexham, UK

\subsection{6/bmjspcare-2018-hospiceabs.77}

'Compassionate Communities help to reduce isolation and loneliness and bring a sense of belonging into what is sometimes a disconnected society.' (Abel \& Kingston, 2018).

Nightingale House Hospice in Wrexham, North Wales covers a diverse catchment area including urban, coastal and rural communities, and includes areas of significant deprivation (Jones, Atenstaedt \& Charles, 2014).

During 2015, following the pilot of a day unit outreach service within a local, rural community, the hospice supported the community to develop a compassionate communities befriending project, and this was followed by two further befriending groups in other communities within our catchment area.

Our presentation describes a service evaluation undertaken with our volunteers from these three compassionate communities groups to identify the motivation for volunteers to become befrienders, their own social circumstances and households and how to improve the establishment of compassionate communities from the perspective of the volunteers delivering the service. The presentation identifies the enthusiasm from the volunteers to deliver a service to others and also the challenges of ensuring systems to ensure befriender safety are in place. The presentation concludes by demonstrating how the feedback from volunteers has impacted on the training delivered, in a demonstration of co-production and the systems for establishing new groups with ten active groups now in development or operation.

\section{Generating research, knowledge and outcomes}

\section{P-53 TRANSFORMING PALLIATIVE CARE SERVICE THROUGH DATA COLLECTION, ANALYSIS AND SHARING}

Karolyn Hallam. Isabel Hospice, Welwyn Garden City, UK

10.1136/bmispcare-2018-hospiceabs.78
Background How is data used to really understand the service that we provide, help target service delivery and assist the development of a clinical strategy to enhance care provision?

Aims Review historical data for gaps in service provision. Identify meaningful data to inform hospice strategy and vision of supporting more people, earlier. Monitor, benchmark our progress and share with staff.

Method We undertook a review of historical data and health needs analysis to identify gaps in service provision. Analysis showed a diverse palliative service user population with patients being referred late in their diagnosis and a small cohort of non-cancer patient referrals, highlighting a gap in specialist palliative intervention. With OACC measures fully embedded in clinical practice, we asked how patient reported outcomes could be used to improve patient care and reflected on IPOS scores quarterly, which showed patients scoring highly in relation to the psychological impact of their illness. We introduced specialist link nurses and expanded our day services to encourage early referrals; a daily morning community meeting to identify, discuss and prioritise those patients with an unstable phase of illness and increased provision of psychological and spiritual support in our family support team.

Results Increased non-cancer referrals into the hospice from $19 \%$ to $40 \%$; reduction of cancer patients dying within one month of referral from $39 \%$ to $35 \% ; 10 \%$ reduction in the percentage of patients scoring severely or overwhelmingly for psychological concerns at the end of spell of care; 18 day average for a patient to move from unstable to stable phase following CNS intervention.

Conclusion Clinical data acts as a vital tool for identifying where action may be needed to address gaps in care delivery. Sharing these outcomes with clinical managers and staff helps everyone to understand the importance of accurate and meaningful data.

\section{P-54 TRANSFORMING SPECIALIST PALLIATIVE CARE WITH DATA}

Duncan Newberry, Collins Okakwu, Laura Myers. Sue Ryder, Nettlebed, UK

\subsection{6/bmispcare-2018-hospiceabs.79}

Background Interprofessional working is vital in the delivery of specialist palliative care (Dawson, 2007); both between and within organisations. Funding bodies require ever stronger evidence before and after gifting monies, and technology is showing potential for the identifying patients in need of palliative care (Tanuseputro, 2017). Use of data has proved problematic in palliative care, leading some to highlight the importance of making the most of routine data (Hanratty, Goldacre, Griffith, et al., 2008).

Aims To evaluate how data are being generated, gathered and used in different areas of the hospice, and the role of technology in this (Nwosu, Collins \& Mason, 2018).

Methods A Clinical Support Analyst position has been created to collate data. To demonstrate the range of educator activity, the Clinical Practice Facilitator (CPF) has been using an app (WonderApps AB, 2018) to collate data about his time division. Registrations onto courses are made through an electronic booking system (Eventbrite). Education evaluation data is collated electronically and brought 